\title{
Actors of the base excision repair play: how well do we know the credits?
}

\author{
Dmitry O. Zharkov \\ Novosibirsk State University, \\ SB RAS Institute of Chemical Biology \\ and Fundamental Medicine, \\ Novosibirsk, Russia \\ dzharkov@niboch.nsc.ru
}

\author{
Anton V. Endutkin, \\ ICBFM, Novosibirsk, Russia \\ aend@niboch.nsc.ru \\ Evgeniia A. Diatlova, \\ ICBFM, Novosibirsk, Russia \\ jannie.lapt@gmail.com
}

Anna V. Yudkina

Novosibirsk State University,

ICBFM, Novosibirsk, Russia

ayudkina@niboch.nsc.ru

Alexander V. Popov,

ICBFM, Novosibirsk, Russia

avpopov@niboch.nsc.ru

\begin{abstract}
Base excision repair (BER) is a pathway responsible for removal of small non-bulky DNA lesions from genomes of all living organisms. BER is initiated by one of several DNA glycosylases that excise the damaged base and is continued by apurinic/apyrimidinic endonucleases, DNA polymerases and DNA ligases. Although the whole BER pathway in both human and $E$. coli cells operates with 20-30 polypeptides, recent finding from other organisms suggest the existence of BER proteins that are not found in wellcharacterized model organisms. Even the homologs of known BER proteins may form families clearly separate from the biochemically characterized ones, and have different functions. Moreover, BER proteins often consist of a universally conserved catalytic core and accessory domains specific for a certain clade. Here, several examples of newly discovered bacterial DNA glycosylases are presented. Evolution of substrate and reaction specificity in BER enzymes is analyzed. The functions of noncatalytic domains or tails of BER proteins are discussed.
\end{abstract}

Keywords - DNA damage, DNA repair, base excision repair, DNA glycosylases, protein structure, protein evolution.

Motivation and Aim

Motivation

Base excision repair (BER) is a pathway responsible for removal of small non-bulky DNA lesions from genomes of all living organisms. BER is initiated by one of several DNA glycosylases that excise the damaged base and is continued by apurinic/apyrimidinic endonucleases, DNA polymerases and DNA ligases. Although the whole BER pathway in both human and E. coli cells operates with 20-30 polypeptides, recent finding from other organisms suggest the existence of BER proteins that are not found in well-characterized model organisms.

Aim

We aimed to mine sequence databases for homologs of known BER proteins in order to find polypeptides and/or their domains forming families clearly separate from the biochemically characterized ones, and to characterize their functions biochemically.

\section{Methods}

We have used Conserved Domain Database [1] to obtain consensus sequences of all BER proteins and used them as queries in BLAST [2] to recover all homologous protein sequences from the NCBI RefSeq database [3]. The sequences were aligned and distance trees were built using Clustal Omega [4]. Coding sequences for Flp1, Flp2, Flp3a, Flp3b, Flp4, SauFpg1, SauFpg2, SauMutY, Ulp2, and Ulp3 proteins were made by total gene synthesis and cloned into $\mathrm{pET}$ series plasmids for expression. The proteins were overproduced and purified by a combination of metal affinity chromatography, ion exchange chromatography, and gel filtration. Substrate specificity of the enzymes was studied using modified oligonucleotides essentially as described for other DNA glycosylases $[5,6]$. X-ray data were collected at BNL National Synchrotron Light Source II. The protein structures were built by molecular replacement using Phaser [7], Coot [8], Phenix [9], Refmac [10], and BUSTER [11]. Molecular dynamic studies were done in Amber 11 [12] employing ff99SB force field with parmbsc 0 corrections $[13,14]$.

\section{Results}

All sequences bearing homology to the known members of helix-two-turn-helix (H2TH) DNA glycosylases (Fpg, Nei, NEIL1, NEIL2, NEIL3, MMH) could be grouped in twelve families, five of which had none biochemically characterized members. To establish substrate specificity and enzymatic mechanisms of these Flp proteins (Fpg-Like Proteins), we have synthesized coding sequences of Flp1 from Streptomyces coelicolor, Flp2 from Xanthomonas campestris, Flp3a and Flp3b from Bacteroides thetaiotaomicron, and Flp4 from Salinibacter ruber, and purified the proteins. The enzymes were mostly Nei/NEIL-like, removing oxidized pyrimidines from DNA and generating a significant fraction of $\beta$-elimination product, unlike Fpg that predominantly yields products of $\beta, \delta$-elimination. Flp3a and Flp3b are different from most $\mathrm{H} 2 \mathrm{TH}$ glycosylases in having $\mathrm{N}$-terminal nucleophilic Ile and Lys, respectively, instead of Pro. The crystal structure of Flp3b was determined and revealed a closed protein conformation (like Fpg and NEIL1 but unlike Nei), a lesion-binding loop significantly different from other H2TH superfamily members, and a partial electron density corresponding to an unprocessed initiator Met residue. Molecular dynamics was used to obtain the structure of the fully processed Flp3b and analyze its interactions with various damaged bases. Additionally, E. coli Fpg with a series of Nterminal substitutions was analyzed biochemically and revealed that Prol is indeed required for activity in $\mathrm{H} 2 \mathrm{TH}$ glycosylases out of the Flp3 family.

All sequences bearing homology to the known members of uracil-DNA glycosylase superfamily (Ung/UNG, Mug/TDG, SMUG1) could be grouped in nine families, three of which had none biochemically characterized members. To establish substrate specificity and enzymatic mechanisms of these Ulp proteins (Ung-Like Proteins), we have synthesized coding sequences of Ulp2 from Helicobacter hepaticus and Ulp3 from Pseudomonas syringae, and purified the proteins. Both were monofunctional DNA glycosylases, as all known superfamily members. Unexpectedly, Ulp3 turned out to lack uracil-removing activity but efficiently processed DNA with oxidized pyrimidines such as 5-hydroxyuracil and 5,6dihydrouracil. 
Some species contain more than ortholog from a given family of DNA glycosylases. For instance, the reference genome of Staphylococcus aureus (strain NCTC 8325), an important human opportunistic pathogen, possesses one homolog for Fpg, yet many isolates of $S$. aureus and other Staphylococcus species contains another homolog, more closely related to Fpg than to Nei. We have produced and characterized both enzymes, and found them to be essentially Fpg-like. However, another DNA glycosylase, MutY, which in $E$. coli and humans counteracts the mutagenic action of 8oxoguanine (oxoG), was different in S. aureus: it excised guanine much better than oxoG.

E. coli MutY and its human homolog, MUTYH, possess a C-terminal domain important for the preferential excision of oxoG. This domain is similar to NUDIX superfamily proteins, which hydrolyze a number of mononucleotide substrate; in particular, E.coli MutT and human MTH1 are pyrophosphatases converting oxodGTP to oxodGMP and preventing its incorporation into DNA. Interestingly, in the static crystal structure of MutY, oxoG interacts with the NUDIX domain in an entirely different manner than in MutT and MTH1, and the oxoG interactions with MutT and MTH1 are also different [15], while kinetic data suggest that the early stages of oxoG recognition by MutY occur in a MutT/MTH1like conformation. All NUDIX sequences could be grouped in 54 families. Comparison of sequences and computer simulation of structures and their interactions with oxoG showed that MutY C-terminal domain is closer to human MTH1; a putative early recognition pocket with a set of specific enzyme-oxoG interactions was revealed in MutY.

Apurinic/apyrimidinic (AP) endonucleases are important enzymes catalyzing the next step of BER after base excision. They cleave DNA at AP sites, and also have a $3{ }^{\prime} \rightarrow 5^{\prime}-$ exonuclease activity, 3'-phosphodiesterase, and 3'phosphatase activity. The major human AP endonuclease, APEX1, belongs to the exonuclease-endonucleasephosphatase superfamily, and the homologs of APEX1 can be grouped in five families, some of which include enzymes with preferential 3'-end processing function. An analysis of sequence conservation and structure allowed us to single out the residues that are responsible for 3 '-processing rather than AP endonuclease activity. Evolution of non-catalytic domains of APEX1-like enzymes shows that this group of polypeptides is very modular, combining the DNA repair function with redox sensing, recombination, and likely other, still undefined functions. The N-terminal tail of human APEX1, in addition to its redox function, was needed to form oligomers on DNA, which might be required for APEX1 interaction with the rest DNA repair machinery and with transcription factors.

\section{ACKNOWLEDGMENT}

Supported by RFBR (20-04-00554-a) and RSF (17-1401190).

\section{REFERENCES}

[1] Marchler-Bauer A. et al. (2015) CDD: NCBI's conserved domain database. Nucleic Acids Res. 43(D1):D222-D226.

[2] Altschul S.F. et al. (1997) Gapped BLAST and PSI-BLAST: A new generation of protein database search programs. Nucleic Acids Res. 25(17):3389-3402.

[3] O’Leary N.A. et al. (2016) Reference sequence (RefSeq) database at NCBI: Current status, taxonomic expansion, and functional annotation. Nucleic Acids Res. 44(D1):D733-D745.

[4] Sievers F. et al. (2011) Fast, scalable generation of high-quality protein multiple sequence alignments using Clustal Omega. Mol. Syst. Biol. 7:539.

[5] Tchou J. et al. (1994) Substrate specificity of Fpg protein: Recognition and cleavage of oxidatively damaged DNA. J. Biol. Chem. 269(21):15318-15324

[6] Zharkov D.O. et al. (2000) Substrate specificity and reaction mechanism of murine 8-oxoguanine-DNA glycosylase. J. Biol. Chem. 275(37):28607-28617.

[7] McCoy A.J. et al. (2007) Phaser crystallographic software. J. Appl. Crystallogr. 40(4):658-674.

[8] Emsley P. et al. (2010) Features and development of Coot. Acta Crystallogr. D Biol. Crystallogr. 66(4):486-501.

[9] Zwart P.H. et al. (2008) Automated structure solution with the PHENIX suite. Methods Mol. Biol. 426:419-435.

[10] Murshudov G.N. et al. (2011) REFMAC5 for the refinement of macromolecular crystal structures. Acta Crystallogr. D Biol. Crystallogr., 67(4):355-367.

[11] Smart O.S. et al. (2012) Exploiting structure similarity in refinement: Automated NCS and target-structure restraints in BUSTER. Acta Crystallogr. D Biol. Crystallogr., 68(4):368-380.

[12] Case D.A. et al. (2010) AMBER 11. University of California, San Francisco.

[13] Hornak V. et al. (2006) Comparison of multiple Amber force fields and development of improved protein backbone parameters. Proteins. 65(3):712-725.

[14] Pérez A. et al. (2007) Refinement of the AMBER force field for nucleic acids: Improving the description of $\alpha / \gamma$ conformers. Biophys. J. 92(11):3817-3829.

[15] Yudkina A.V. et al. (2019) Reading and misreading 8-oxoguanine, a paradigmatic ambiguous nucleobase. Crystals, 9(5):269. 